\title{
Genetic and Environmental Contributions to Stability in Adult Obsessive Compulsive Behavior
}

\author{
Nuno R. Zilhão, ${ }^{1,2}$ Dirk J. A. Smit, ${ }^{2}$ Anouk den Braber, ${ }^{2}$ Conor V. Dolan, ${ }^{2,3}$ Gonneke Willemsen, ${ }^{2,4}$ \\ Dorret I. Boomsma, ${ }^{2,4}$ and Danielle C. Cath ${ }^{1,5}$ \\ ${ }^{1}$ Department of Clinical and Health Psychology, Utrecht University, Utrecht, the Netherlands \\ ${ }^{2}$ Department of Biological Psychology, VU University, Amsterdam, the Netherlands \\ ${ }^{3}$ Department of Psychological Methods, University of Amsterdam, Amsterdam, the Netherlands \\ ${ }^{4}$ EMGO+ Institute for Health and Care Research, VU University Medical Centre, Amsterdam, the Netherlands \\ ${ }^{5}$ Altrecht Academic Anxiety Center, Utrecht, the Netherlands
}

\begin{abstract}
This study investigates the relative contribution of genetic and environmental factors to the stability of obsessive-compulsive $(\mathrm{OC})$ symptoms in an adult population-based sample. We collected data from twin pairs and their siblings, using the Padua Inventory Revised Abbreviated, from the population-based Netherlands Twin Register (NTR) in $2002(n=10.134)$ and $2008(n=15.720)$. Multivariate twin analyses were used to estimate the stability of $\mathrm{OC}$ symptoms as a function of genetic and environmental components. OC symptoms were found to be highly stable, with a longitudinal phenotypic correlation of 0.63 . Longitudinal broad sense heritability was found to be $56.0 \%$. Longitudinal correlations for genetic ( $r=0.58$ for additive, $r=1$ for non-additive genetic factors) and non-shared environment $(r=0.46)$ reflected stable effects, indicating that both genes and environment are influencing the stability of $\mathrm{OC}$ symptoms in adults. For the first time, evidence is reported for non-additive genetic effects on the stability of OC symptoms. In conclusion, this study showed that $\mathrm{OC}$ symptoms are highly stable across time in adults, and that genetic effects contribute mostly to this stability, both in an additive and non-additive way, besides non-shared environmental factors. These data are informative with respect to adult sample selection for future genetic studies, and suggest that gene-gene interaction studies are needed to further understand the dominance effect found in this study.
\end{abstract}

- Keywords: obsessive-compulsive symptoms, obsessive compulsive disorder, stability, heritability, twin study, dominance, non-additive genetic effects, genetic epidemiology

Obsessive-compulsive disorder (OCD) is characterized by intrusive, unwanted thoughts, and repetitive behaviors performed in a ritualized fashion (APA, 1994). It has a lifetime prevalence of $0.5-2.0 \%$ (APA, 1994), and it is, among all anxiety disorders, recognized by the World Health Organization (WHO) as a leading cause for non-fatal, illnessrelated disability, affecting mostly individuals between 15 and 44 years of age (WHO, 2007). Quality of life is seriously impaired in OCD, more so than, for instance, in depression (Srivastava et al., 2011). Various longitudinal clinical studies have established that in contrast to children/adolescents in whom OC symptoms seem to remit somewhat more often (Fernández de la Cruz et al., 2013; Leckman et al., 2009; Micali et al., 2010; Stewart et al., 2004), in adults, OC symptoms tend to be more stable with respect to symptom dimensions, with probability estimates of full remission between $17 \%$ and $27 \%$, and of partial remission of between $22 \%$ and $53 \%$ in the course of up to 40 years, depending on study methodology (prospective vs. retrospective), and country of origin (Alonso et al., 2001; Eisen et al., 2013; Mancebo et al., 2014; Orloff et al., 1994; Reddy et al., 2005; Skoog \& Skoog, 1999; Steketee et al., 1999). Specifically, in patients who only experience partial treatment response, symptoms recur in up to $70 \%$ of patients within 5 years of follow-up (Eisen et al., 2013). Longitudinal studies on OC symptoms are far more scarce in epidemiological than in clinical cohorts, both in children and in adults (Angst et al., 2004; Fineberg et al., 2013).

RECEIVED 25 October 2014; ACCEPTED 3 November 2014. First published online 18 December 2014.

ADDRESS FOR CORRESPONDENCE: Nuno R. Zilhão, Department of Biological Psychology, VU University, van de Boechorststraat 1, 1081 BT Amsterdam, the Netherlands. E-mail: n.rodrigueszilhaonogueira@vu.nl 
Only two studies examined OCD and OC symptoms in a community cohort. The Zurich community cohort study followed a group of adolescents for 30 years (Angst et al., 2004; Fineberg et al., 2013). This study indicated that over this 30 -year period, at age 50 in more than one third of the sample, OC symptoms had not remitted. Fullana et al. (2007) examined the temporal stability of OC dimensions over a period of 2 years in undergraduate students, and found no significant changes in OC symptom scores between baseline and follow-up, except for the occurrence of obsessions (Fullana et al., 2007). These data provide a longitudinal perspective on OCD and OC symptoms in adults, but do not address the etiology for the observed stability, of which still little is known.

Several family-based studies have indicated a familial basis for OCD, with increased frequencies of OC symptoms in first-degree relatives (Do Rosario-Campos et al., 2005; Pauls et al., 1995). Population-based twin family studies have shown that variation in OC symptoms is heritable (Do Rosario-Campos et al., 2005; Hudziak et al., 2004; Iervolino et al., 2011; Pauls et al., 1995) with somewhat lower heritabilities in adults $\left(\mathrm{h}^{2}\right.$ ranging between $\left.0.30-0.40\right)$ than in children $\left(\mathrm{h}^{2}\right.$ between $0.45-0.58$ for 12-year-old twins and 0.55 for 6-year-old twins; Hudziak et al., 2004; Bolton et al., 2009), and little support for common environment and non-additive genetic effects.

Some recent studies have extended these findings by introducing a longitudinal twin design. A first study at the Dutch Twin register (van Grootheest et al., 2007a) longitudinally examined the stability of OC symptoms in twins aged 7, 10, and 12 years old. The twins were measured on OC symptoms using the Obsessive Compulsive Scale of the Child Behavior Checklist. The twin design allows stability to be attributed to either genetic or environmental factors. Van Grootheest et al. (2007a) reported an average phenotypic longitudinal correlation of 0.5 across a 5 -year period in children between ages 7, 10, and 12. Genetic factors explained a substantial part (between 35\% [father ratings] and $51 \%$ [mother ratings]) of this OC symptom stability. Fully in line with these findings, Bolhuis et al. (2014) used OC symptom data from a longitudinal cohort of adolescent twins and siblings $(n=2,651$; the Genesis $12-19$ study; mean age 15 years), and a cross-sectional sample of adult twins ( $n=4,920$; mean age $=55$ years $)$ to explore genetic and environmental relationships between $\mathrm{OC}$ and depressive symptoms both cross-sectionally and longitudinally (Bolhuis et al., 2014). Within the adolescent sample, covariance (B) between OC symptoms at time points 1 and 2 (with mean 25 months interval between measurements) was 0.48 (CI between $0.42-0.56$ ), indicating substantial stability over time.

A previous genetic epidemiological study has been conducted in adult twins within the Dutch Twin Register on OC symptoms (van Grootheest et al., 2009). Twins (av- erage age at baseline: 17.8 years) were measured at four time points between 1991 and 2002, using two different measurement scales - the YASR-OCS (Nelson et al., 2001) at the first three time points and the Padua Inventory Revised Abbreviated (PI-R ABBR; Cath et al., 2008a) at time point 4 . The correlation across time ranged between 0.39 and 0.60 between sequential measurement occasions, and the longitudinal heritability was calculated to be around $40 \%$. However, the stability measures of OC symptoms and the etiology of that variance may have been obscured by the use of different measurement instruments at different time points. It is reasonable to expect that different scales tag different sources of variation, thus leading to an underestimation of stability and the proportion of that estimation to be attributed to genetic factors.

Therefore, the aim of the present study was to extend the findings of the van Grootheest et al. (2009) study, overcoming the methodological weakness of the previous study (different instruments used at different time points to measure OC symptoms); moreover, including siblings in the analyses to explore OC symptoms over a period of 6 years, and to determine the genetic and environmental contributions to stability of OC symptoms in a large sample of adult twins and their siblings. OC symptoms were measured in 2002 and in 2008 at a subsequent wave of collection within the NTR, with a large proportion of the subjects overlapping with the 2002 wave.

\section{Materials and Methods}

\section{Participants and Procedure}

The data for this study were collected as part of longitudinal survey studies of the NTR. Since 1991, every 2-3 years, twins and their families are assessed and receive surveys by mail, with questionnaires about health, personality, and lifestyle. For the present analysis, we analyzed the OC data collected at the 2002 and 2008 wave of collection, corresponding to Surveys 6 and 8 . The total sample consisted of 20,376 individuals from 7,812 different families. In Survey $6,10,134$ individuals responded, and 15,720 responded to Survey 8. Longitudinal data were available for 5,478 individuals. Twin participants with incomplete data on zygosity $(N=41$ for complete pairs, and $N=170$ for incomplete pairs) were excluded from the analysis. This study has been approved by the Medical Ethical Committee of the VU Medical Centre Amsterdam. In the genetic analysis, we included a maximum of four siblings (two brothers and two sisters) per family. Table 1 gives an overview of the total number of participants. The number of complete twin pairs included in the study by zygosity is given in Table 2 . Zygosity was assessed by a questionnaire using items on physical similarity, blood group and DNA polymorphisms (Willemsen et al., 2013). 
TABLE 1

Number of Participants Included in Genetic Analysis

\begin{tabular}{|c|c|c|c|c|c|c|}
\hline & \multicolumn{3}{|c|}{ Survey 6} & \multicolumn{3}{|c|}{ Survey 8} \\
\hline & Male & Female & Male + female & Male & Female & Male + female \\
\hline \multicolumn{7}{|l|}{ Twins } \\
\hline$N$ & 1,398 & 2,987 & 4,385 & 2,286 & 5,163 & 7,449 \\
\hline Age & 32.27 & 32.46 & & 33.65 & 34.05 & \\
\hline Age $S D$ & 11.48 & 11.15 & & 14.92 & 14.51 & \\
\hline \multicolumn{7}{|l|}{ Siblings } \\
\hline$N$ & 552 & 843 & 1,395 & 629 & 1,069 & 1,698 \\
\hline Age & 35.01 & 34.62 & & 38.97 & 37.77 & \\
\hline Age $S D$ & 13.06 & 11.13 & & 14.72 & 13.59 & \\
\hline
\end{tabular}

TABLE 2

Number of Twins by Zygosity

\begin{tabular}{lccr}
\hline & Survey 6 & Survey 8 \\
Twin zygosity & $N$ twins $(N$ complete pairs) & $N$ twins $(N$ complete pairs) & Total number of unique twins \\
\hline Monozygotic males (MZM) & $635(231)$ & $1,017(354)$ & 1,306 \\
Dizygotic males (DZM) & $360(102)$ & $623(182)$ & 800 \\
Monozygotic females (MZF) & $1,588(625)$ & $2,714(1,047)$ & 3,260 \\
Dizygotic females (DZF) & $847(298)$ & $1,412(458)$ & 1,763 \\
Dizygotic opposite sex (DOS) & $955(287)$ & $1,683(418)$ & 2,151 \\
\hline
\end{tabular}

\section{Phenotype Measures}

For both Surveys 6 and 8, OC symptom scores were measured with the Padua Inventory abbreviated (PI-R ABBR; Cath et al., 2008). This 12-item questionnaire has been derived from the Padua Inventory-Revised (PI-R), a widely used 41-item self-report inventory on OC symptoms, with item ratings between 0-4, and five subscales (washing, checking, rumination, precision, and impulses; Sanavio, 1988; van Oppen, 1992). The 12-item PI-R ABBR contains two items on each subscale; items have been chosen based on the highest factor loadings in a previous validation study (Van Oppen et al., 1995), and one item has been added to the rumination and impulses subscales.

\section{Genetic Modeling and Testing}

Genetic epidemiological twin studies are based on comparing the different degrees of family relatedness between family members, to estimate the relative contribution of the genetic and the environmental components to a trait. Monozygotic (MZ) twins share (nearly) all their genes, whereas dizygotic (DZ) twins, just like non-twin siblings, share on average half of their segregating genes. In quantitative genetics, the total phenotypic variance is decomposed into variance components due to genetic $(G)$, shared environmental (C) and non-shared environmental (E) factors. C reflects the common environmental effects shared by family members and $\mathrm{E}$ the non-shared environmental influences, that is, the unique environmental component for an individual. The genetic variance can be additive $(\mathrm{A})$, reflecting the additive effect of different alleles, or non-additive (dominance; D), indicating interaction between alleles. The comparison between $\mathrm{MZ}$ and $\mathrm{DZ}$ twin and sibling correlations provides a first impression of the relative contributions of each component. The greater the phenotypic similarity between MZ twins when compared to DZ twins and non-twin siblings, the more the variance of the trait is explained by genetic factors.

An MZ correlation that is the double the DZ correlation is indicative of additive genetic influences. DZ correlations higher than half of the MZ correlations indicate the role of shared environmental factors, while DZ correlations that are less than half of the MZ correlations indicate genetic non-additive effects (dominance). All genetic analyses were carried out with the use of Structural Equation Modeling as implemented in OpenMx (Boker et al., 2011). A saturated model (with input of a $6 \times 6$ data matrix of two twins, two brothers, and two sisters for questionnaire data) was used to estimate familial correlations, to test for sex differences and for the effect of age on the OC symptom scores. Next, to evaluate the stability of the PI-R ABBR scores between the two time points, a bivariate saturated model (with input of a $12 \times 12$ data matrix of 2 twins, 2 brothers and 2 sisters, each with two time-point measures), was fitted. These models estimate variances and covariances within and between $\mathrm{MZ}$ and DZ twins and between twins and siblings, and specify all correlations between family members. Next, a genetic model (ADE model) was fitted to the data to estimate the relative proportion of $\mathrm{A}, \mathrm{D}$, and $\mathrm{E}$ in $\mathrm{OC}$ symptom scores, as the twin correlations indicated an $\mathrm{ADE}$ was more appropriate than an ACE model. The comparison between different models (e.g., ADE vs. AE) was done by means of likelihood-ratio tests. The negative log likelihood $(-2 \mathrm{LL})$ of a more restricted model is subtracted from the -2LL of a more general model. This generates a statistic 

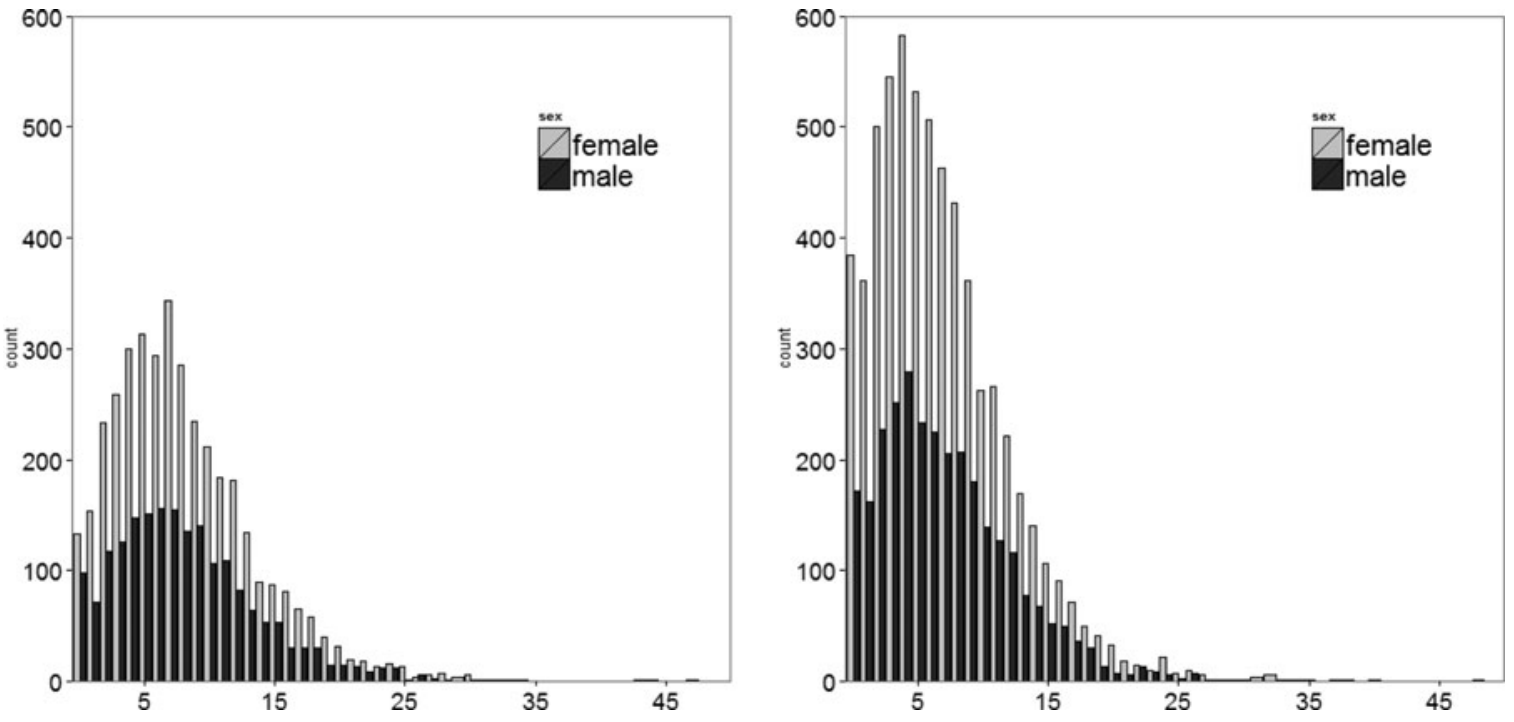

\section{FIGURE 1}

Distribution of OC symptom scores, before transformation of the data, in both survey 6 (left), and 8 (right).
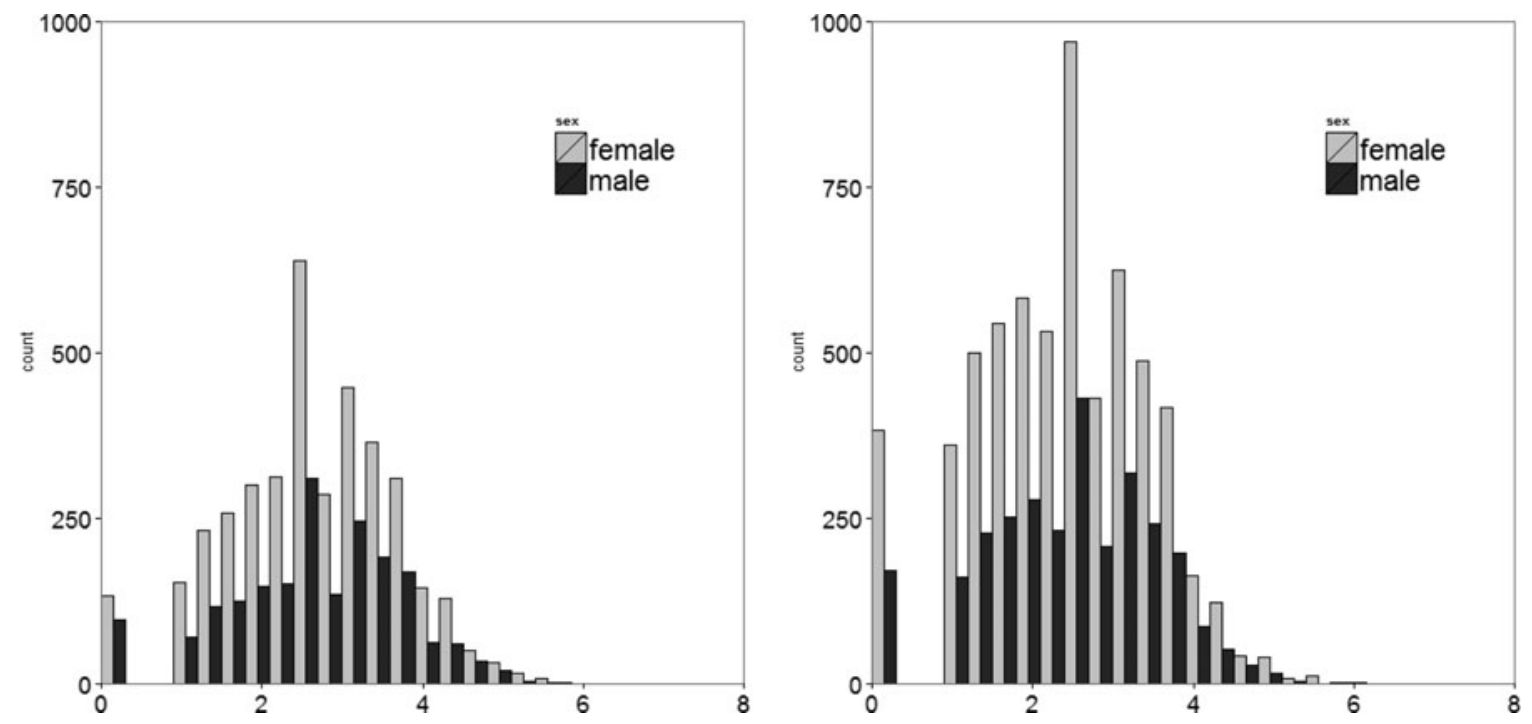

\section{FIGURE 2}

Distribution of OC symptom scores in both survey 6 (left), and 8 (right), after square-root transformation of the data.

that is distributed as a $\chi^{2}$ distribution with degrees of free$\operatorname{dom}(d f)$ equal to the difference in the number of estimated parameters in the two models. The more parsimonious constrained model is selected if the $\chi^{2}$ yields a nonsignificant $p$-value. An alpha of 0.01 was set as threshold for significance.

\section{Results}

Longitudinal data were available for 5,478 individuals (2,048 males, 3,430 females). Their average age was 33.0 years $(S D=11.5)$ at Survey 6 , and 34.7 years $(S D=14.6)$ at Survey 8. The correlation between the two surveys was 0.63 . The effect of age on OC symptom scores was a drop of 0.03 per year for both Surveys 6 and 8 .

Since the distribution of OC symptom scores was skewed ( kkewness $=1.10$ in Survey 6, and skewness $=1.22$ in Survey 8 ; Figure 1), scores were transformed using square root transformation. The graph in Figure 2 plots the distribution of OC symptom scores, after transformation of the data (skewness $=-0.315$ in Survey 6, and skewness $=-0.254$ in Survey 8).

The estimates for familial correlations are given in Tables 3 and 4, for Surveys 6 and 8 respectively. These are 
TABLE 3

Familial Correlations Estimated From Maximum Likelihood in Survey 6

\begin{tabular}{llrr}
\hline Type of relationship & \multicolumn{1}{l}{$95 \% \mathrm{Cl}$} & $n$ pairs \\
\hline MZ twins & 0.398 & $0.371-0.471$ & 856 \\
MZ males & 0.414 & $0.305-0.507$ & 231 \\
MZ females & 0.400 & $0.335-0.460$ & 625 \\
Male relatives & 0.227 & $0.125-0.321$ & 446 \\
DZ male & 0.253 & $0.084-0.400$ & 102 \\
Brother-male twin & 0.196 & $0.062-0.316$ & 292 \\
Brother-brother & 0.296 & $-0.014-0.524$ & 52 \\
Female relatives & 0.150 & $0.079-0.220$ & 1,135 \\
DZ female & 0.207 & $0.084-0.318$ & 298 \\
Sister-female twin & 0.134 & $0.0176-0.191$ & 732 \\
Sister-sister & 0.058 & $-0.121-0.231$ & 105 \\
Female-male & 0.106 & $0.038-0.171$ & 456 \\
DOS & 0.228 & $0.099-0.342$ & 287 \\
Sister-brother & -0.025 & $-0.213-0.168$ & 169 \\
\hline
\end{tabular}

TABLE 4

Familial Correlations Estimated From Maximum Likelihood in Survey 8

\begin{tabular}{lllr}
\hline Type of relation & $r$ & \multicolumn{1}{l}{$95 \% \mathrm{Cl}$} & $\mathrm{n}$ pairs \\
\hline MZ twins & $\mathbf{0 . 3 9 6}$ & $\mathbf{0 . 3 5 3 - 0 . 4 3 6}$ & $\mathbf{1 , 3 8 5}$ \\
MZ males & 0.385 & $0.301-0.460$ & 354 \\
MZ females & 0.407 & $0.356-0.455$ & 1,047 \\
Male relatives & $\mathbf{0 . 1 2 4}$ & $\mathbf{0 . 0 2 7}-0.219$ & $\mathbf{4 9 1}$ \\
DZ male & 0.209 & $0.060-0.337$ & 182 \\
Brother-male twin & 0.041 & $-0.092-0.172$ & 256 \\
Brother-brother & 0.278 & $-0.201-0.564$ & 53 \\
Female relatives & 0.106 & $\mathbf{0 . 0 4 8}-0.164$ & $\mathbf{1 , 3 3 7}$ \\
DZ female & 0.114 & $0.026-0.198$ & 458 \\
Sister-female twin & 0.091 & $0.012-0.167$ & 777 \\
Sister-sister & 0.144 & $-0.080-0.345$ & 102 \\
Female-male & 0.168 & $\mathbf{0 . 1 1 0 - 0 . 2 2 2}$ & $\mathbf{5 8 7}$ \\
DOS & 0.222 & $0.125-0.311$ & 418 \\
Sister-brother & -0.013 & $-0.219-0.199$ & 169 \\
\hline
\end{tabular}

\section{TABLE 5}

Cross Twin-Cross Time Correlations

\begin{tabular}{lll}
\hline Type of relationship & $r$ & $95 \% \mathrm{Cl}$ \\
\hline MZ twins & $\mathbf{0 . 3 6 7}$ & $\mathbf{0 . 3 2 7 - 0 . 4 0 6}$ \\
MZ males & 0.400 & $0.324-0.468$ \\
MZ females & 0.359 & $0.308-0.400$ \\
DZ twins/siblings & $\mathbf{0 . 1 1 9}$ & $0.867-0.151$ \\
DZ male/brothers & 0.130 & $0.090-0.169$ \\
DZ female/sisters & 0.084 & $0.036-0.131$ \\
DOS/sister-brothers & 0.157 & $0.083-0.228$ \\
\hline
\end{tabular}

given for $\mathrm{MZ}$ and $\mathrm{DZ}$ twins, sib-sib and twin-sib pairs, conditional on sex. Monozygotic males (MZM) and monozygotic females (MZF) correlations are moderate, and both sib-sib and sib-twin correlations, at Survey 8, are smaller than half of the MZ correlations. The observed patterns in familial correlations, when comparing MZ correlations with all other first-degree relatives, suggest that genetic factors may play a role in individual differences for OC symptoms. We observed a high stability for OC symptoms across time with correlations of 0.629 for within individual measurements. Cross twin-cross time correlations (Table 5) represent the correlations between the scores in one twin at one time point, and its co-twin at another time point.
First- and second-born twin correlations are constrained to be equal to second-born and first-born correlations. Cross twin-cross time correlations were estimated for males and females separately, being slightly higher in males (MZ CTCT: $R_{\text {male }}=0.400, R_{\text {female }}=0.359$; DZ/Sibling CTCT: $\left.R_{\text {male }}=0.130, R_{\text {female }}=0.084\right)$. When estimated and compared between $\mathrm{MZ}$ and $\mathrm{DZ}$ twin pairs, the results suggest that the stability in OC symptom scores is predominantly due to genetic factors.

Model fitting analyses are displayed in Supplementary Table S1 for Surveys 6 and 8. There were no differences in variances across zygosity or between all pairs, and no differences in variances and correlations between the sexes. In both surveys, for twin-sib pairs, correlations could be constrained to be equal; equality assumptions were tested separately for males (Table S1, model 3 compared to model 2), for females (Table S1, model 4 compared to model 3), and for opposite-sex relatives (Table S1, model 5 compared to model 4). Gender differences were also tested for equality and constrained to be equal (Table S1, model 6 compared to model 5). The correlation between MZM and MZF could also be constrained to be equal, estimated at 0.398 $(95 \% \mathrm{CI}=0.371-0.471)$ for Survey 6 , and $0.399(95 \% \mathrm{CI}=$ 0.356-0.440) for Survey 8 (Table S1, model 7 compared to model 6).

Working from a fully saturated model, considering all results from model comparisons and the correlations between $\mathrm{MZ}$ and $\mathrm{DZ}$ twins, we decided to next fit a genetic ADE model to the data. The relative contributions $\mathrm{D}$ could not be constrained to zero $\left(\chi^{2}=11.464, d f=3, p=0.009\right)$, and consequently the best fitting model was the ADE. Figure 3 shows the unstandardized estimates of the ADE model. This allows one to compute the relative contributions of both $\mathrm{A}$, $\mathrm{D}$, and $\mathrm{E}$ for each time point, given on the diagonal of Table 6.

The results show that for both time points the contributions of $A$ and D to OC symptoms are around 0.2. Because the contribution of $\mathrm{D}$ could not be dropped from the model, this results in a broad sense heritability estimate (both A and D components) of 0.420 and 0.418 for surveys 6 and 8 respectively. For non-shared environmental influences this was around 0.58 for both time points. The off-diagonal estimated in Table 6 gives the results of the decomposition of the phenotypic stability between the two time points. The result show that $56 \%$ of the stability for OC symptoms is due to genetic factors (both A and D components). Non-shared environmental factors had a moderate contribution to the stability of $44 \%$. Finally, longitudinal correlations were calculated, indicating the relative overlap of genetic and environmental effects between the two time points. The additive genetic correlation was estimated at 0.58 from the ADE model. This value indicates a moderately high overlap for the genetic influences between both time points. For the $\mathrm{D}$ component, a correlation of 1 was obtained, indicating a perfect overlap for non-additive effects between the two time 


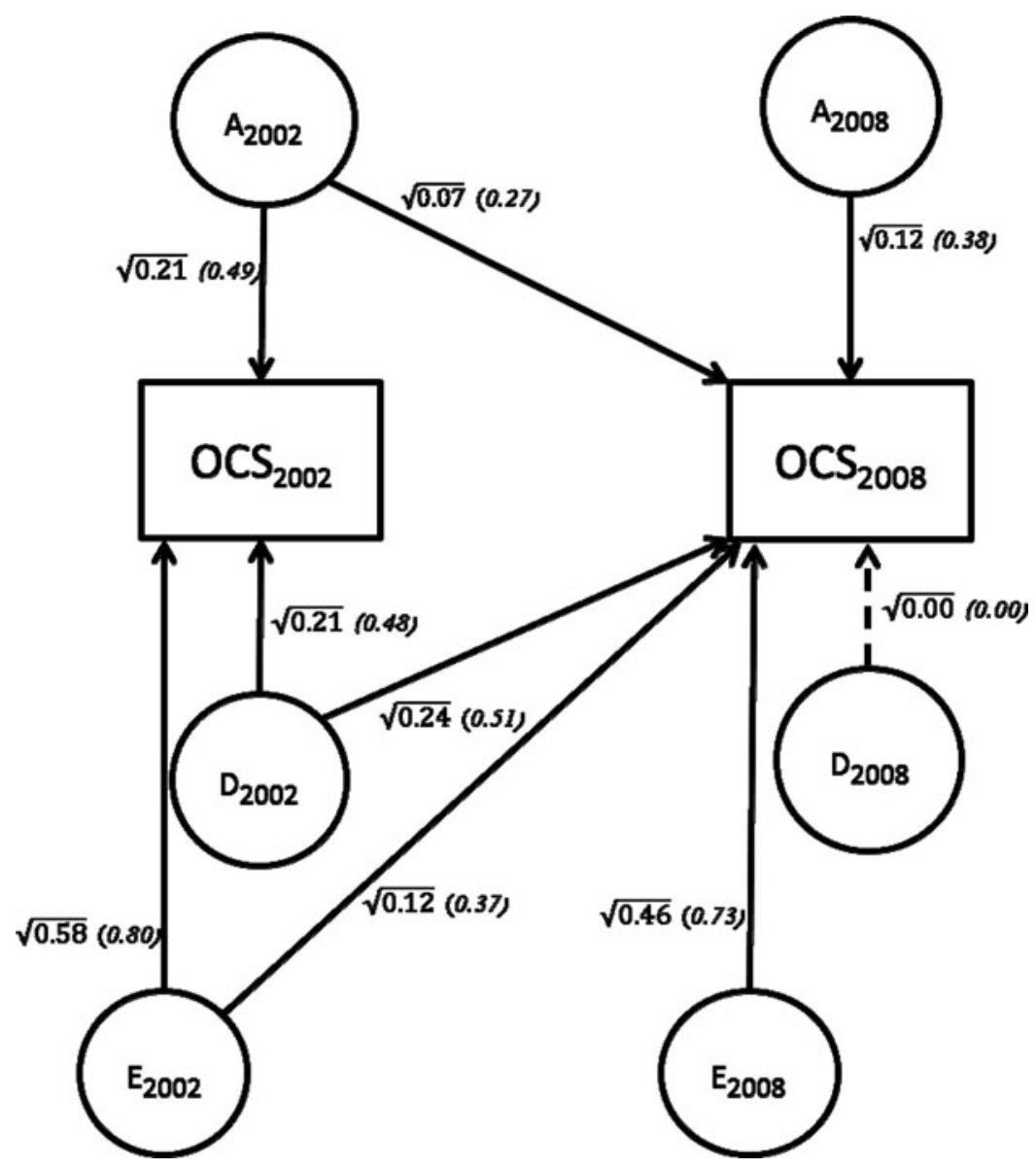

\section{FIGURE 3}

Standardized and unstandardized (italics) estimates of the final ADE model for OC symptoms at the two time points. Rectangles represent observed variables at the two time points, OCS 2002 (survey 6) and OCS 2008 (survey 8). Circles represent the latent factors A (additive genetic influences), D (dominant genetic influences) and E (non-shared environmental influences). The dashed line represents a non-significant path. The values represent the loadings of each observed variable in the latent factors.

TABLE 6

Relative Contributions of Additive Genetic and Non-Shared Environmental Influences within Time (Diagonal) and Across Time (Off Diagonal) for PI-R ABBR

\begin{tabular}{|c|c|c|c|c|c|c|}
\hline & \multicolumn{2}{|c|}{$\begin{array}{l}\text { Additive genetic } \\
\text { effects }(A)\end{array}$} & \multicolumn{2}{|c|}{$\begin{array}{c}\text { Non-additive genetic } \\
\text { effects (D) }\end{array}$} & \multicolumn{2}{|c|}{$\begin{array}{c}\text { Non-shared environmental } \\
\text { effects }(E)\end{array}$} \\
\hline & Survey 6 & Survey 8 & Survey 6 & Survey 8 & Survey 6 & Survey 8 \\
\hline Survey 6 & 0.212 & - & 0.208 & - & 0.580 & - \\
\hline Survey 8 & 0.194 & 0.187 & 0.365 & 0.231 & 0.441 & 0.582 \\
\hline
\end{tabular}

points, and that no new ' $D$ ' is involved at a later stage. For non-shared environmental factors, the correlation was 0.46 .

\section{Discussion}

To our knowledge, this is the first genetic epidemiological study in twin adults assessing the longitudinal genetic and environmental contributions to the stability of OC symptoms using the PI-R ABBR questionnaire at multiple time points. First, in slight contrast to the previous longitudinal study in children (van Grootheest et al., 2007b), we found no quantitative sex differences in average OC scores or heritability estimates in this adult sample, with the same additive genetic factors influencing both males and females. In line with previous cross-sectional studies in adolescents at different time points and in adults using the YASR-OCS (van Grootheest et al., 2007b; van Grootheest et al., 2008), no evidence for a special twin environment was found, since 
correlations could be equated across zygosities and between twins and siblings. Heritability estimates at each time point were also in line with what is described in the literature. The values for the broad sense heritability (both A and D components) at both time points were at around $42 \%$, and the remaining variance was due to unique environment. In a recent article addressing the shared genetic and environmental contributions to both OC symptoms and hoarding, with the same adult twin data from the NTR 2008 wave of collection as used in these analyses, the authors found heritabilities of $40 \%$ for OC symptoms (Mathews et al., 2014). In an earlier report on the 2002 wave of data collection, van Grootheest et al. (2009) found heritability rates of $38 \%$ and $44 \%$, for males and females respectively. All these studies in adults found no contribution of shared environment. Only in one study in children, a small contribution of shared environment has been found at age 12 (van Grootheest et al., 2008), suggesting differences in environmental architecture underlying stability of OC symptoms in children versus adults.

We found the OC symptoms to be highly stable, with a longitudinal phenotypic correlation of 0.63 across a 6year time interval. Van Grootheest et al. (2009), using the same 2002 wave of data collection with the Padua-R-ABBR in 2002 as an end point, and the 1991, 1995, and 1997 data waves that used the Young Adult Self-Report Obsessive Compulsive Scale (YASR-OCS) as starting points, found longitudinal phenotypic correlations of around 0.2 (for the 11-year time interval) and 0.4 (for a 5-year time interval). These different results could be explained by the fact that in that study the stability of OC symptoms was calculated across different measurement scales. Furthermore, the patterns in cross twin-cross time correlations $(r=0.367$ for MZ twins, $r=0.119$ for DZ twins/siblings) show that the stability is due to both genetic and non-shared environmental factors (Table 6). This suggests that the same genes are expressed across time and are influencing OC symptoms, and also that a substantial amount of $\mathrm{E}$ reflects stable effects. Collectively, these sets of results show that in adults, data from men and women, as well as twins and non-twins of different ages, can be combined, which is particularly beneficial in molecular genetic studies, where combining data will result in an increase in power to detect underlying genetic effects.

Of interest are the results regarding the decomposition of the longitudinal phenotypic variance. Previously it was reported that for children, common environmental influences explain part of the stability (around 40\%; van Grootheest et al., 2007a), unlike what happens in adult family members, who generally do not share the same household any more with their respective co-twin. Instead, it was shown that in adults, about $70 \%$ of the stability was due to additive genetic factors (van Grootheest et al., 2009). Here, we found no common environmental influences for the stability of OC symptoms but we have, however, presented for the first time new evidence for contributions of unique environmental influences in adults, and these influences correlate substantially across time $(r=0.46)$. This relatively high contribution of non-shared environment to the stability of OC symptoms (off-diagonals in Table 6) indicates that unstable variance such as measurement error (timepoint specific variance) cannot account for its variation. Therefore, individual experiences may have a relevant impact on the stability of OC symptom in adults. The detrimental influence of early-life experiences might persist and influence OC symptomatology into adulthood. One study specifically addressed the influence of unique and shared environmental factors in developing (or protecting against) OC symptoms, by comparing scores between highly concordant and highly discordant MZ twins (Cath et al., 2008). The comparison of highly within-discordant pairs indicated some important influencing life events, among which the most relevant appeared to be past experiences of sexual assault. These possible risk factors were more highly associated with OC symptoms in high-scoring discordant MZ pairs than in high-scoring concordant MZ pairs, thus pointing to the relevance of individual experiences in childhood in developing OC symptomatology. It appears then, that once OC symptoms are acquired in early adulthood, stability tends to be higher and more genetically mediated than in children.

For the first time, we observed significant non-additive genetic effects. Dominant effects have not been observed before for OC symptoms, even in the same subject sample. Non-additive variance encompasses all forms of genetic factors with a non-linear effect such as epistasis/gene-gene interaction and dominant effects (Mather, 1974). This may have unexpected consequences for molecular genetics approaches, because most genome-wide technologies, such as genome-wide association analysis and genome-wide complex trait analysis (GCTA; Yang et al., 2011), primarily assume additive genetic variance. These studies may have been underpowered by not including dominance in the linear regression models. Random effects modeling using GCTA to explain phenotypic variance (Yang et al., 2010) may not be able to provide SNP-based heritability estimates for all genetic effects and lead to 'missing heritability', since modeling non-additive effects requires very large sample sizes (Yang et al., 2013).

In sum, although our results for the broad sense heritability estimates (all genetic effects involved) are in line with what has been described before for narrow sense heritability (Davis et al., 2013; van Grootheest et al., 2007b; van Grootheest et al., 2009), they do indicate that the genetic etiology of OC symptoms and their stability in time may be more complex than previously thought, and that some differences occur with respect to its underlying etiologies between children and adults. Molecular and genome-wide studies as well as twin studies could, in the future, include dominance effects in the linear regression, 
and/or take epistatic effects/gene-gene interactions into account.

\section{Acknowledgments}

We are grateful to the twins and siblings for their participation. This project has been financed by FP7-People-2012ITN, project: TS-Eurotrain, grant number 316978; BBR Foundation (NARSAD) 21668; ZonMW (Addiction) Projectnummer: 31160008; and European Research Council (ERC-230374).

\section{Supplementary Material}

To view supplementary material for this article, please visit http://dx.doi.org/(10.1017/thg.2014.77).

\section{References}

Alonso, P., Pujol, J., Cardoner, N., Benlloch, L., Deus, J., ... Vallejo, J. (2001). Right prefrontal repetitive transcranial magnetic stimulation in obsessive-compulsive disorder: A double-blind, placebo-controlled study. American Journal of Psychiatry, 158, 1143-1145.

American Psychiatric Association (APA) (1994). Diagnostic and statistical manual of mental disorders (4th ed.). Washington, DC: Author.

Angst, J., Gamma, A., Endrass, J., Goodwin, R., Ajdacic, V., Eich, D., ... Rössler, W. (2004). Obsessive-compulsive severity spectrum in the community: Prevalence, comorbidity, and course. European Archives of Psychiatry and Clinical Neuroscience, 254, 156-164.

Boker, S., Neale, M., Maes, H., Wilde, M., Spiegel, M., Brick, T., ... Fox, J. (2011). OpenMx: An open source extended structural equation modeling framework. Psychometrika, 76, 306-317.

Bolhuis, K., McAdams, T. A., Monzani, B., Gregory, A. M., Mataix-Cols, D., Stringaris, A., ... Eley, T. C. (2014). Aetiological overlap between obsessive-compulsive and depressive symptoms: A longitudinal twin study in adolescents and adults. Psychological Medicine, 44, 1439-1449.

Bolton, D., Rijsdijk, F., Eley, T. C., O’Connor, T. G., Briskman, J., \& Perrin, S. (2009). Normative childhood repetitive routines and obsessive compulsive symptomatology in 6-yearold twins. Journal of Child Psychology and Psychiatry, and Allied Disciplines, 50, 1139-1146.

Cath, D. C., van Grootheest, D. S., Willemsen, G., van Oppen, P., \& Boomsma, D. I. (2008). Environmental factors in obsessive-compulsive behavior: Evidence from discordant and concordant monozygotic twins. Behavior Genetics, 38, $108-120$.

Davis, L. K., Yu, D., Keenan, C. L., Gamazon, E. R., Konkashbaev, A. I., Derks, E. M., ... Scharf, J. M. (2013). Partitioning the heritability of Tourette syndrome and obsessive compulsive disorder reveals differences in genetic architecture. PLoS Genetics, 9, e1003864.

Do Rosario-Campos, M. C., Leckman, J. F., Curi, M., Quatrano, S., Katsovitch, L., Miguel, E. C., ... Pauls, D. L. (2005). A family study of early-onset obsessive-compulsive disorder. American Journal of Medical Genetics - Neuropsychiatric Genetics, 136B, 92-97.

Eisen, J. L., Sibrava, N. J., Boisseau, C. L., Mancebo, M. C., Stout, R. L., Pinto, A., ... Rasmussen, S. A. (2013). Fiveyear course of obssesive-compulsive disorder: Predictors of remission and relapse. Journal of Clinical Psychiatry, 74, 233-239.

Fernández de la Cruz, L., Barrow, F., Bolhuis, K., Krebs, G., Volz, C., Nakatani, E., ... Mataix-Cols, D. (2013). Sexual obsessions in pediatric obsessive-compulsive disorder: Clinical characteristics and treatment outcomes. Depression and Anxiety, 30, 732-740.

Fineberg, N. A., Hengartner, M. P., Bergbaum, C., Gale, T., Rössler, W., \& Angst, J. (2013). Lifetime comorbidity of obsessive-compulsive disorder and sub-threshold obsessive-compulsive symptomatology in the community: Impact, prevalence, socio-demographic and clinical characteristics. International Journal of Psychiatry in Clinical Practice, 17, 188-196.

Fullana, M. A., Tortella-Feliu, M., Caseras, X., Taberner, J., Torrubia, R., \& Mataix-Cols, D. (2007). Temporal stability of obsessive-compulsive symptom dimensions in an undergraduate sample: A prospective 2-year follow-up study. Behavior Modification, 31, 815-824.

Hudziak, J. J., Van Beijsterveldt, C. E. M., Althoff, R. R., Stanger, C., Rettew, D. C., Nelson, E. C., ... Boomsma, D. I. (2004). Genetic and environmental contributions to the child behavior checklist obsessive-compulsive scale: A cross-cultural twin study. Archives of General Psychiatry, 61, 608-616.

Iervolino, A. C., Rijsdijk, F. V, Cherkas, L., Fullana, M. A., \& Mataix-Cols, D. (2011). A multivariate twin study of obsessive-compulsive symptom dimensions. Archives of General Psychiatry, 68, 637-644.

Leckman, J. F., Bloch, M. H., \& King, R. A. (2009). Symptom dimensions and subtypes of obsessive-compulsive disorder: A developmental perspective. Dialogues in Clinical Neuroscience, 11, 21-33.

Mancebo, M. C., Boisseau, C. L., Garnaat, S. L., Eisen, J. L., Greenberg, B. D., Sibrava, N. J., ... Rasmussen, S. A. (2014). Long-term course of pediatric obsessivecompulsive disorder: 3 years of prospective follow-up. Comprehensive Psychiatry, 55, 1498-1504.

Mather, K. (1974). Non-allelic interaction in continuous variation of randomly breeding populations. Heredity, 32, 414419.

Mathews, C. A., Delucchi, K., Cath, D. C., Willemsen, G., \& Boomsma, D. I. (2014). Partitioning the etiology of hoarding and obsessive-compulsive symptoms. Psychological Medicine, 44, 2867-2876.

Micali, N., Heyman, I., Perez, M., Hilton, K., Nakatani, E., Turner, C., ... Mataix-Cols, D. (2010). Long-term outcomes of obsessive-compulsive disorder: Follow-up of 142 children and adolescents. The British Journal of Psychiatry, 197, 128-134.

Nelson, E. C., Hanna, G. L., Hudziak, J. J., Botteron, K. N., Heath, A. C., \& Todd, R. D. (2001). Obsessivecompulsive scale of the child behavior checklist: 
Specificity, sensitivity, and predictive power. Pediatrics, 108, e14.

Orloff, L. M., Baer, L., Ivanjack, L., Battle, M. A., Buttolph, M. L., \& Jenike, A. (1994). Long-term follow-up of 85 patients with obsessive-compulsive disorder. American Journal of Psychiatry, 151, 441-442.

Pauls, D. L., Alsobrook, J. P., Goodman, W., Rasmussen, S., \& Leckman, J. F. (1995). A family study of obsessivecompulsive disorder. The American Journal of Psychiatry, 152, 76-84.

Reddy, Y. C. J., D’Souza, S. M., Shetti, C., Kandavel, T., Deshpande, S., Badamath, S., ... Singisetti, S. (2005). An 11- to 13-year follow-up of 75 subjects with obsessivecompulsive disorder. The Journal of Clinical Psychiatry, 66, 744-749.

Sanavio, E. (1988). Obsessions and compulsions: The padua inventory. Behaviour Research and Therapy, 26, 169-177.

Skoog, G., \& Skoog, I. (1999). A 40-year follow-up of patients with obsessive-compulsive disorder. Archives of General Psychiatry, 56, 121-127.

Srivastava, S., Bhatia, M. S., Thawani, R., \& Jhanjee, A. (2011). Quality of life in patients with obsessive compulsive disorder: A longitudinal study from India. Asian Journal of Psychiatry, 4, 178-182.

Steketee, G., Eisen, J., Dyck, I., \& Warshaw, M. (1999). Predictors of course in obsessive-compulsive disorder. Psychiatry Research, 89, 229-238.

Stewart, S. E., Geller, D. A., Jenike, M., Pauls, D., Shaw, D., \& Mullin, B. (2004). Long-term outcome of pediatric obsessive-compulsive disorder: A meta-analysis and qualitative review of the literature. Acta Psychiatrica Scandinavica, 110, 4-13.

Van Grootheest, D. S., Bartels, M., Cath, D. C., Beekman, A. T., Hudziak, J. J., \& Boomsma, D. I. (2007a). Genetic and environmental contributions underlying stability in childhood obsessive-compulsive behavior. Biological Psychiatry, 61, 308-315.

Van Grootheest, D. S., Bartels, M., van Beijsterveldt, C. E. M., Cath, D. C., Beekman, A. T., Hudziak, J. J., . . Boomsma,
D. I. (2008). Genetic and environmental contributions to self-report obsessive-compulsive symptoms in Dutch adolescents at ages 12,14, and 16. Journal of the American Academy of Child and Adolescent Psychiatry, 47, 11821188.

Van Grootheest, D. S., Cath, D., Hottenga, J. J., Beekman, A. T., \& Boomsma, D. I. (2009). Genetic factors underlie stability of obsessive-compulsive symptoms. Twin Research and Human Genetics, 12, 411-419.

Van Grootheest, D. S., Cath, D. C., Beekman, A. T., \& Boomsma, D. I. (2007b). Genetic and environmental influences on obsessive-compulsive symptoms in adults: A population-based twin-family study. Psychological Medicine, 37, 1635-1644.

Van Oppen, P. (1992). Obsessions and compulsions: Dimensional structure, reliability, convergent and divergent validity of the Padua Inventory. Behaviour Research and Therapy, 30, 631-637.

Van Oppen, P., Hoekstra, R. J., \& Emmelkamp, P. M. (1995). The structure of obsessive-compulsive symptoms. Behaviour Research and Therapy, 33, 15-23.

Willemsen, G., Vink, J. M., Abdellaoui, A., den Braber, A., van Beek, J. H. D. A., Draisma, H. H. M., ... Boomsma, D. I. (2013). The adult Netherlands twin register: Twenty-five years of survey and biological data collection. Twin Research and Human Genetics, 16, 271-281.

World Health Organization (WHO) (2007). World Health Statistics 2007. Geneva: Author.

Yang, J., Benyamin, B., McEvoy, B. P., Gordon, S., Henders, A. K., Nyholt, D. R., ... Visscher, P. M. (2010). Common SNPs explain a large proportion of the heritability for human height. Nature Genetics, 42, 565-569.

Yang, J., Lee, S. H., Goddard, M. E., \& Visscher, P. M. (2011). GCTA: A tool for genome-wide complex trait analysis. American Journal of Human Genetics, 88, 76-82.

Yang, J., Lee, S. H., Goddard, M. E., \& Visscher, P. M. (2013). Genome-wide complex trait analysis (GCTA): Methods, data analyses, and interpretations. Methods in Molecular Biology, 1019, 215-236. 